Conference topic: Technology of luminescent materials

\title{
Fabrication of submicron-sized oxide phosphors and their spectroscopic properties
}

\author{
E. Zych, A. Walasek, J. Trojan-Piegza, A. Kossek \\ Faculty of Chemistry, University of Wrocław, 14 F. Joliot-Curie Street, 50383 Wrocław, Poland \\ zych@wchuwr.chem.uni.wroc.pl
}

In our report we will present a few synthesis techniques, which may be routinely used to fabricate nanoparticulate or submicron-sized powder phosphor materials. We will focus on procedures, which may be easily scalable and which allow making powders of low agglomeration or aggregation and which are energy-efficient.

Among others we will report on making powders of garnets, YAG:Pr, YAG:Eu, LuAG:Pr by means of molten salts technique giving particles of almost spherical shape and sizes in the range of $200-500 \mathrm{~nm}$, as seen in Figure 1. What is the most special in the case of this fabrication technique is an extremely low temperature necessary to convert a stoichiometric mixture of the metal nitrates into crystalline garnets. The process may be effectively performed below $400{ }^{\circ} \mathrm{C}$ even. However, the best results may be obtained carrying out the synthesis around $500{ }^{\circ} \mathrm{C}$. The classical ceramic synthesis requires temperatures in the range of $1500-1700{ }^{\circ} \mathrm{C}$. To the best of our knowledge, only pressure-assisted synthesis techniques allow to reduce the temperatures of the process to a few hundred centigrade.

We will also present synthesis method based on homogeneous precipitation of solids from water solutions with or without the use of surfactants. We will discuss the influence of $\mathrm{SO}_{4}^{2-}$ ions addition on the properties of the final products, especially on particles agglomeration. We shall discuss the applicability of these preparation techniques to fabrication of powders for X-ray detection.

The description of the various fabrication methods will be associated with presentation of the powders X-ray diffraction analysis and TEM/SEM images. These will prove the

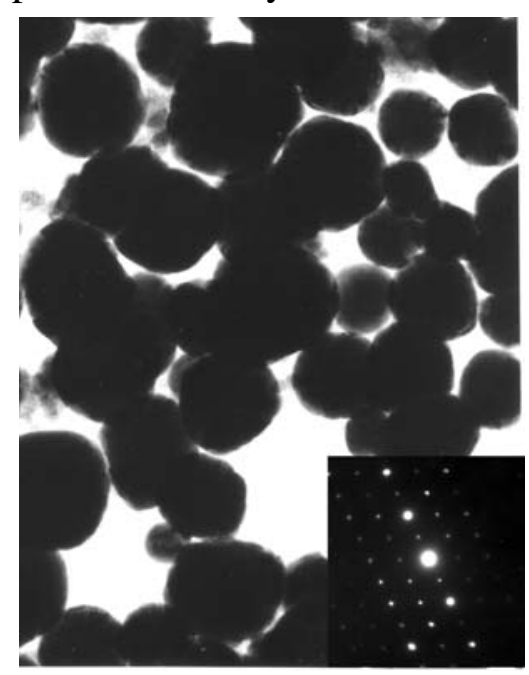
nonagglomerated microstructure and crystalline character of the products.

A part of the talk we will devote to present some spectroscopic results characterizing the luminescent properties of some of the products. Among others we shall discuss results of experiments performed with the use of synchrotron radiation (Hasylab, Superlumi Station in Hamburg).

Figure 1. Microstructure of a YAG:Eu powder synthesized using molten salts technique and SAED pattern of a single grain. 